\title{
Article
}

\section{Interstitial Lung Disease and Anti-Myeloperoxidase Antibodies: Not a Simple Association}

\author{
Marco Sebastiani $^{1, *(D)}$, Fabrizio Luppi ${ }^{2} \mathbb{D}$, Gianluca Sambataro ${ }^{3} \mathbb{D}$, Diego Castillo Villegas ${ }^{4}$, Stefania Cerri ${ }^{5}$, \\ Paola Tomietto ${ }^{6}$, Giulia Cassone ${ }^{1,7}$, Marialuisa Bocchino ${ }^{8}$, Belen Atienza-Mateo ${ }^{9} \mathbb{D}$, Paolo Cameli $^{10} \mathbb{D}$, \\ Patricia Moya Alvarado 4 , Paola Faverio ${ }^{2}$, Elena Bargagli 10 ${ }^{(D)}$, Carlo Vancheri ${ }^{3}$, Miguel A. Gonzalez-Gay ${ }^{9,11} \mathbb{D}$, \\ Enrico Clini ${ }^{5}$, Carlo Salvarani ${ }^{1,12}$ and Andreina Manfredi ${ }^{1}$
}

1 Rheumatology Unit, Azienda Ospedaliera Policlinico di Modena, University of Modena and Reggio Emilia, 41121 Modena, Italy; giulia.cassone@unimore.it (G.C.); carlo.salvarani@unimore.it (C.S.); Andreina.Manfredi@gmail.com (A.M.)

2 Department of Medicine and Surgery, University of Milano Bicocca, Respiratory Unit, San Gerardo Hospital, ASST di Monza, 20900 Monza, Italy; fabrizio.luppi@unimib.it (F.L.); paola.faverio@unimib.it (P.F.)

3 Regional Referral Centre for Rare Lung Diseases, A.O.U. "Policlinico-Vittorio Emanuele", Department of Clinical and Experimental Medicine, University of Catania, 95123 Catania, Italy; dottorsambataro@gmail.com (G.S.); vancheri@unict.it (C.V.)

4 Respiratory Department, Hospital de la Santa Creu i Sant Pau, 08041 Barcelona, Spain; DCastillo@santpau.cat (D.C.V.); pmoyaa@santpau.cat (P.M.A.)

5 Respiratory Unit, University of Modena and Reggio Emilia, 41121 Modena, Italy; stefania.cerri@unimore.it (S.C.); enrico.clini@unimore.it (E.C.)

6 Rheumatology Unit, University of Trieste, 34127 Trieste, Italy; paola.tomietto@asuits.sanita.fvg.it

check for updates

Citation: Sebastiani, M.; Luppi, F.; Sambataro, G.; Castillo Villegas, D.; Cerri, S.; Tomietto, P.; Cassone, G.; Bocchino, M.; Atienza-Mateo, B.; Cameli, P.; et al. Interstitial Lung Disease and Anti-Myeloperoxidase Antibodies: Not a Simple Association. J. Clin. Med. 2021, 10, 2548. https:// doi.org/10.3390/jcm10122548

Academic Editor: Gasparri Roberto

Received: 10 May 2021

Accepted: 7 June 2021

Published: 9 June 2021

Publisher's Note: MDPI stays neutral with regard to jurisdictional claims in published maps and institutional affiliations.

Copyright: (C) 2021 by the authors. Licensee MDPI, Basel, Switzerland. This article is an open access article distributed under the terms and conditions of the Creative Commons Attribution (CC BY) license (https:/ / creativecommons.org/licenses/by/ $4.0 /)$.
7 PhD Program in Clinical and Experimental Medicine, University of Modena and Reggio Emilia, 41121 Modena, Italy

8 Department of Clinical Medicine and Surgery, Federico II University Hospital, 80131 Naples, Italy; marialuisa.bocchino@unina.it

9 Department of Rheumatology, Hospital Universitario Marqués de Valdecilla, IDIVAL, 39011 Santander, Spain; mateoatienzabelen@gmail.com (B.A.-M.); miguelaggay@hotmail.com (M.A.G.-G.)

10 Respiratory Diseases Unit, Department of Medical Sciences, Surgery and Neurosciences, Siena University, 53100 Siena, Italy; paolocameli88@gmail.com (P.C.); bargagli2@gmail.com (E.B.)

11 Cardiovascular Pathophysiology and Genomics Research Unit, School of Physiology, Faculty of Health Sciences, University of the Witwatersrand, Johannesburg 2094, South Africa

12 Rheumatology Unit, Irccs Arcispedale Santa Maria Nuova, Azienda Unità Sanitaria Locale-IRCCS di Reggio Emilia, 42124 Reggio Emilia, Italy

* Correspondence: marco.sebastiani@unimore.it 
Keywords: interstitial pneumonia; idiopathic pulmonary fibrosis; vasculitis; rheumatic diseases; anti-myeloperoxidase antibodies

\section{Introduction}

Anti-neutrophil cytoplasmic antibodies (ANCA) are autoantibodies specific for antigens located in the cytoplasmic granules of neutrophils and lysosomes of monocytes [1]. Myeloperoxidase (MPO) and proteinase 3 (PR3) are the two main autoantigen targets. ANCA are generally detected in multisystemic diseases, namely ANCA-associated vasculitides (AAV), characterized by necrotizing vasculitis typically involving small vessels [2]. After the first description in 1990, the association between interstitial lung disease (ILD) and AAV was confirmed in 1994 in a Japanese study that reported a 43\% prevalence of ILD in MPO-ANCApositive patients with collagen vascular disease and or glomerulonephritis [3].

On the other side, an increasing number of authors reported the presence of ANCA in patients with idiopathic pulmonary fibrosis (IPF), with a prevalence ranging from 1 to $35 \%$, mainly in the Japanese population [4-6]. Anti-MPO antibodies are the most frequent ANCA subtype described in these patients. Moreover, ANCA conversion may occur in up to $11 \%$ of patients during the IPF course [7]. The role of anti-MPO antibodies in patients with idiopathic interstitial pneumonia (IIPs) remains unclear. The occurrence of vasculitis, mainly microscopic polyangiitis (MPA), has been described in a minority of cases. Indeed, only $7-23 \%$ of ANCA-positive subjects subsequently developed a clinically overt vasculitis during the follow-up, mainly limited to patients with anti-MPO antibodies [8,9].

Conflicting data have been reported about the prognostic role of ANCA antibodies in IPF patients. Some authors reported no difference in survival, while others observed a poorer prognosis in ANCA-positive IPF patients, regardless of the appearance of vasculitis $[5,7,8,10,11]$.

This multicenter, retrospective study aimed to investigate the clinical, serological, and radiological features, as well as the prognosis of anti-MPO-positive ILD patients.

\section{Materials and Methods}

\subsection{Study Population and Data Collection}

We retrospectively collected the clinical charts of all consecutive anti-MPO-positive patients from 6 Italian and Spanish centers with a special interest in ILD, firstly referred for IIP from 1 January 2015 to 31 December 2019. All patients showed a positivity for anti-MPO antibodies at the time of ILD diagnosis. The search for anti-MPO antibodies was performed by combining indirect immunofluorescence and ELISA using tests with similar specificity and sensitivity in the different centers. Patients with a previous diagnosis of connective tissue disease (CTD) or systemic vasculitis were excluded from the study. The study was conducted according international ethics standard and was approved by the local ethical committee; patients gave their informed consent to be enrolled.

Data about clinical manifestation suggestive of vasculitis or CTD, lung function testing, autoimmunity data, and CT imaging pattern were recorded for each patient at the time of the first and of the last available visit.

Based on previous reports on classification of patterns of pulmonary involvement in chest high-resolution tomography (HRCT), patient chest HRCT findings were categorized into usual interstitial pneumonia (UIP), nonspecific interstitial pneumonia (NSIP), organizing pneumonia (OP), or other patterns that can also be observed in combination [12-14]. All HRCT images were locally reviewed by an expert thoracic radiologist.

Lung function and CT imaging variations were recorded for the observation period available for each patient. An absolute decline $>10 \%$ of the forced vital capacity (FVC) or $>15 \%$ of the lung diffusion capacity of CO from baseline were considered clinically significant [12]. Improvement, worsening, or stability of chest HRCT was evaluated according to the percentage of involvement of the lung parenchyma [15]. Main disease- 
specific treatments, including immune-suppressants and anti-fibrotic agents, were recorded as well. The end of the follow-up was set at the last available visit or when progression to AAV or death occurred. A patient was defined as lost to follow-up when he/she had no follow-up visit or when the follow-up duration was lower than 12 months. At the end of follow-up, all diagnoses were discussed in a multidisciplinary discussion involving rheumatologist and pulmonologist.

Finally, the survival of MPO-positive-ILD patients was compared with that of a reference cohort of 116 Italian age-matched patients with anti-MPO-negative IPF referred to the University of Modena.

\subsection{Statistical Analysis}

Results were expressed as median and interquartile range (IQR) for numerical data, while frequency was reported as number and percentage.

Categorical variables were analyzed by Chi-square test or Fisher's exact test and differences between the means were determined using the Mann-Whitney test for unpaired samples. $p$ values $<0.05$ were considered statistically significant. Statistical analyses were performed using the SPSS statistical software, version 17.0 (SPSS Inc., Chicago, IL, USA) [16].

\section{Results}

Fifty-eight patients were enrolled in the study (F/M 27/31; median age 69 years, IQR 9) and observed for a median follow-up of 39 months (IQR 51). The baseline characteristics of the study population are reported in Table 1.

Table 1. Baseline features of 58 anti-MPO-ILD patients.

\begin{tabular}{|c|c|c|}
\hline & $n$ & $\%$ \\
\hline Sex $M / F$ & $27 / 31$ & $46.6 / 53.4$ \\
\hline Smoking history & 23 & 39.7 \\
\hline \multicolumn{3}{|l|}{ ILD pattern } \\
\hline UIP & 30 & 51.7 \\
\hline NSIP & 11 & 19.0 \\
\hline OP & 4 & 6.9 \\
\hline \multirow[t]{2}{*}{ Mixed pattern } & 13 & 22.4 \\
\hline & Median & IQR \\
\hline Age at ILD diagnosis & 69 & 9 \\
\hline Follow-up duration (months) & 39.0 & 51 \\
\hline FVC baseline (\%) & 84 & 28.25 \\
\hline FVC end follow-up (\%) & 80 & 29 \\
\hline DLCO baseline $(\%)$ & 55 & 27.0 \\
\hline DLCO end follow-up (\%) & 49 & 36.0 \\
\hline
\end{tabular}

Anti-MPO: anti-myeloperoxidase antibodies; ILD: interstitial lung disease; M: males; F: females; UIP: usual interstitial pneumonia; NSIP: nonspecific interstitial pneumonia; OP: organizing pneumonia; FVC: forced vital capacity; DLCO: diffusion lung capacity for CO; IQR: interquartile range.

At the first observation, only nonspecific symptoms and signs of vasculitis or CTD were recorded, namely sicca syndrome and Raynaud's phenomenon, with no evidence of any systemic involvement. Three patients $(3 / 58=5 \%)$ satisfied the criteria for a diagnosis of interstitial pneumonia with autoimmune features (IPAF) $[17,18]$. Low-titer antinuclear antibodies (ANA) were detected in 19 patients $(32.7 \%)$, without significant extractable nuclear antigen (ENA) specificities.

The baseline ventilatory pattern was preserved in the majority of cases, with FVC values $>70 \%$ of those predicted in 45 subjects $(77.6 \%)$. Conversely, gases exchange was impaired with estimated DLCO values $>80 \%$ of predicted limited to seven patients (12.1\%).

Usual interstitial pneumonia was the most frequent radiologic pattern of ILD at presentation, being observed in more than half of patients (51.7\%). An NSIP was recorded 
in 11 cases $(19 \%)$, OP in four $(6.9 \%)$, and other or combined patterns in the remaining $22.4 \%$ of patients.

Overall, five patients had only one visit and were classified as lost to follow-up; the remaining patients (91\%) had a median follow-up period of 39 months (IQR 50). Among these, $22(41.5 \%)$ received at least one immune-suppressive drug, mainly azathioprine or mycophenolate mofetil, whereas $13(24.5 \%)$ were treated with anti-fibrotic drugs, as detailed in Table 2. Finally, 34 patients $(64.1 \%)$ received chronic systemic corticosteroids in five cases in association with anti-fibrotic drugs. Treatment with anti-fibrotic was exclusively limited to patients with a chest CT UIP pattern, while immune-suppressants were used regardless of imaging features.

Table 2. Proposed treatments in 58 anti-MPO-ILD patients.

\begin{tabular}{ccc}
\hline & $n$ & $\%$ \\
\hline Azathioprine & 11 & 20.7 \\
Cyclophosphamide & 4 & 7.5 \\
Mycophenolate & 8 & 15.1 \\
Rituximab & 3 & 5.7 \\
\hline No immunosuppressors & 31 & 58.5 \\
1 immunosuppressor & 19 & 35.8 \\
2 immunosuppressors & 2 & 3.8 \\
3 immunosuppressors & 1 & 1.9 \\
\hline Pirfenidone & 8 & 15.1 \\
Nintedanib & 5 & 9.4 \\
Steroids & 34 & 64.1 \\
$\mathrm{O}_{2}$ supplementation & 18 & 34 \\
\hline
\end{tabular}

Lung function slightly deteriorated over time in the whole study population. Median FVC declined from 84 to $80 \%$ of the predicted value $(p=0.031)$, while predicted DLCO passed from 55 to $49 \%(p<0.001)$.

In more details, a decline in FVC > 10\% was observed in 20 out of 53 patients $(37.7 \%)$, while it improved in eight (15.1\%). A significant reduction in DLCO (>15\%) occurred in 22 patients $(41.5 \%)$, while it was ameliorated in five $(9.4 \%)$. Consistently, disease extension assessed by chest HRCT worsened in 24 patients (45.2\%) and improved in nine (16.9\%).

After a median follow-up period of 27 months (range 12-111), nine out $53(17 \%)$ patients developed a definite $\mathrm{AAV}$, and an additional four cases $(7 \%)$ developed another rheumatic disease, namely Sjogren's syndrome in three cases and rheumatoid arthritis in one (Figure 1).

No differences were observed in baseline features between patients who did or did not develop a rheumatic disease. In particular, sicca syndrome and arthralgias were not predictive of progression to vasculitis or CTD. Only six out of 19 patients $(36 \%)$ with baseline ANA developed a rheumatic disease ( $p$ not significant when compared with ANA-negative patients).

Finally, the median survival time for ILD-MPO patients was 192 months. The overall cohort of anti-MPO-positive ILD patients had a better 52-week survival in comparison with 116 age-matched reference IPF cases $(71.4 \pm 7.5 \%$ vs. $52.4 \pm 5.9 \%$ for anti-MPO-positive ILD patients and IPF, respectively; $p=0.045)$. Conversely, no survival differences with IPF were observed when looking at MPO-positive ILD patients with a chest CT pattern of UIP $(62.4 \pm 12.3 \%$; Figure 2). 


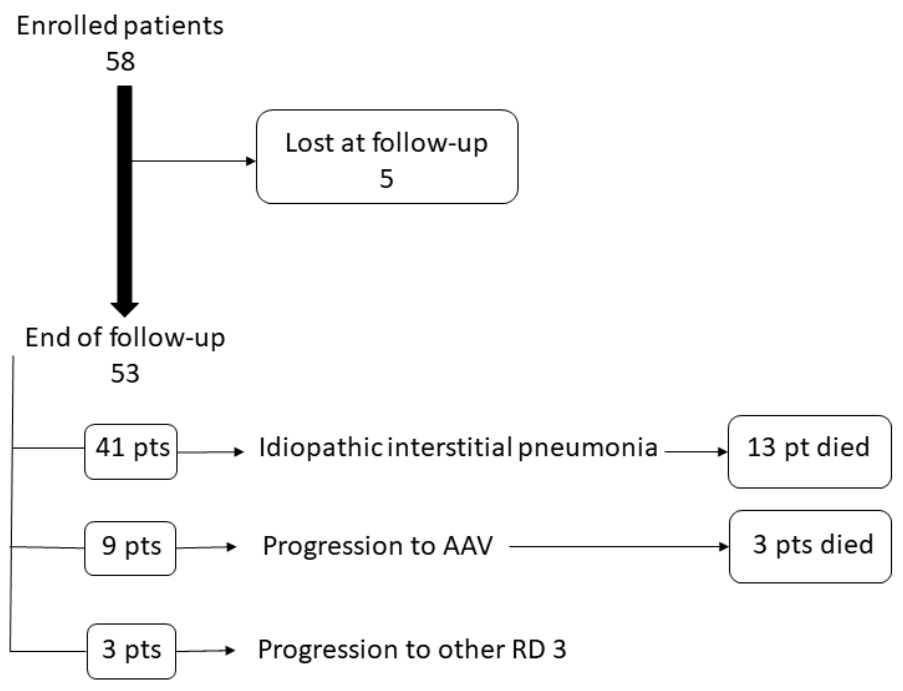

Figure 1. Clinical evolution of patients enrolled in the study.

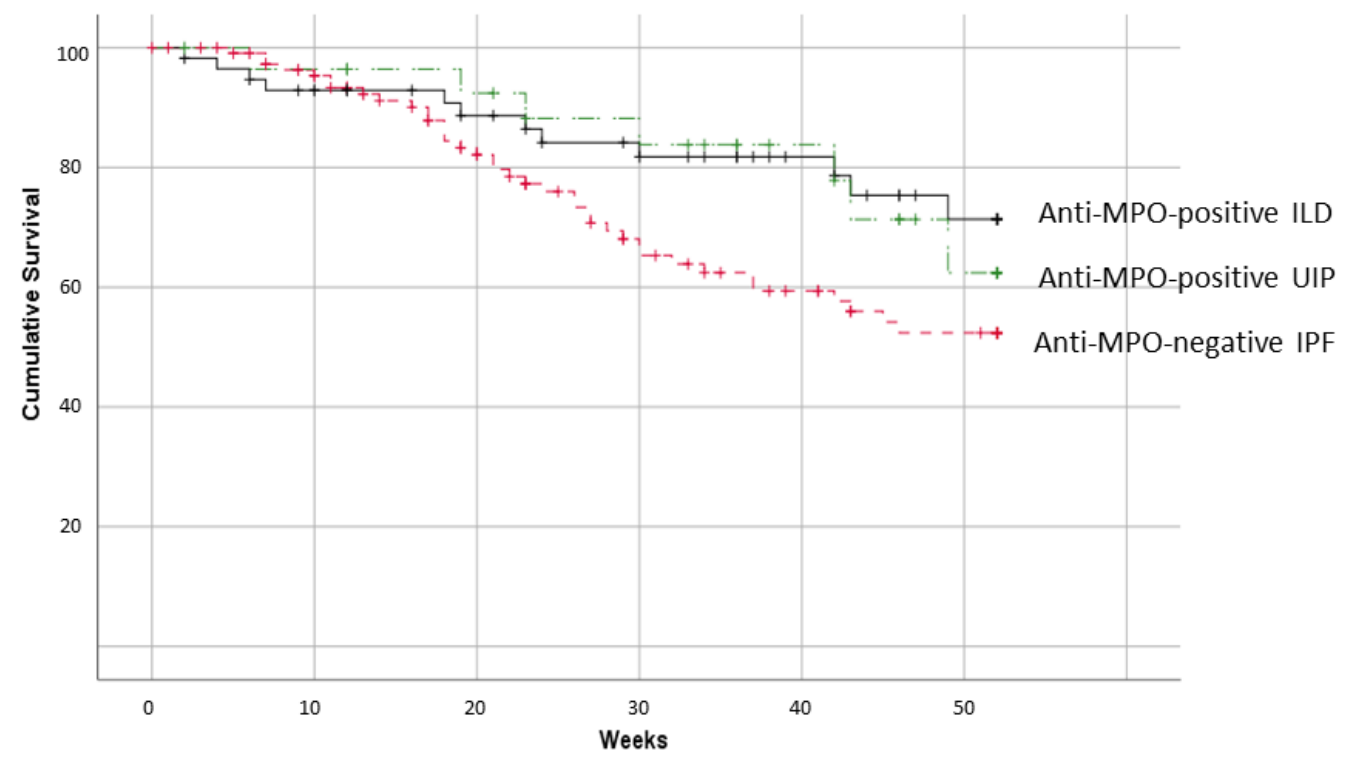

Figure 2. Fifty-two-week survival of patients with anti-MPO, anti-MPO-UIP, and IPF.

\section{Discussion}

In 1999, Becker-Merok et al. described an IPF patient developing glomerulonephritis and necrotizing vasculitis, as well as positivity for p-ANCA antibodies [19].

Afterward, many authors suggested an association between IPF and anti-MPO antibodies, regardless of the occurrence of vasculitis, mainly micro-polyangiitis (MPA) $[4,5,7,8,10,11]$.

In IPF patients, a positivity for ANCA antibodies may be observed in about $5-10 \%$ of cases $[4,5]$. In particular, anti-MPO antibodies have been reported in $4-36 \%$ of patients with idiopathic interstitial pneumonia, including IPF, while the occurrence of MPA has been described in a range from 1.7 to $25.7 \%$ of patients [20,21]. This group of patients might be called "pulmonary limited vasculitis" and considered a phenotypic variant of MPA, as reported by Katsumata et al. [22].

ILD appearance follows the diagnosis of MPA only in a minority of cases. At the same time, in more than $80 \%$, it is diagnosed concurrently or before vasculitis $[3,10,23-26]$, with an interval of time between diagnoses of ILD and vasculitis ranging from a few months to many years. In our population, 13 patients developed a rheumatic disease. Of interest, 
an AAV was only observed in nine patients, MPA in all cases, whereas the other three developed Sjogren's syndrome and the latter rheumatoid arthritis.

Interestingly, in our population, no serological or clinical features were associated with the progression to a rheumatic disease. Few studies described the possible association of anti-MPO antibodies with RA or SS. Anti-MPO antibodies have been described to occur in a low number of RA patients, without any change in clinical and radiologic features of the disease [27], while association with SS is very rare and mainly limited to patients with concurrent AAV [28,29].

Interestingly, in our population, no serological or clinical features were associated with the progression to a rheumatic disease. This is particularly true for patients evolving in MPA. In contrast, in patients developing RA and SS, ANA were positive at baseline, and some clinical manifestations, such as arthralgia and sicca syndrome, were recorded. On the other side, only a few patients with ANA positivity developed a rheumatic disease in the subsequent years. Anyway, a careful clinical and anamnestic evaluation should always be performed to identify patients at risk for evolution in a systemic rheumatic disease. Moreover, a systematic search for complement fractions, renal function, and inflammation indexes, such as C-reactive protein, should be assessed in all patients with anti-MPO antibodies.

For the absence of evidence-based treatment, the therapy proposed to our patients was very heterogeneous, reflecting the single center's experience. Radiological patterns of ILD seem to be the main driver of therapeutic choice; in fact, antifibrotic drugs, namely nintedanib and pirfenidone, have been prescribed in 13 patients, all with UIP pattern. On the other side, administration of immunosuppressants did not appear related to the lung disease features, because this treatment was prescribed regardless of radiological pattern. Moreover, the treatment did not seem to affect lung disease progression. Among nine patients with an improvement of HRCT features, only three had been treated (with azathioprine, mycophenolate mofetil, and pirfenidone, respectively), while an immunosuppressive (10 patients) or antifibrotic (eight patients) therapy was prescribed in patients with disease worsening. Therefore, we can suppose that lung deterioration and severity, more than the ILD pattern, could represent the driver for starting treatment. Of interest, patients initially treated with an immunosuppressant changed their immunosuppressive drug when deterioration of lung function was recorded, but no patients were shifted from immunosuppressive to antifibrotic therapy or vice versa.

While the presence of ILD with a UIP pattern significantly reduces the survival of patients with ANCA-associated vasculitis [30], conflicting data have been reported about the possible influence of ANCA on the survival of IPF patients. In fact, many authors described similar survival rates for patients positive or negative for ANCA [7,8,10,11,22,26], while others suggested a worse prognosis for ANCA-positive patients, mainly anti-PR3 antibodies [19].

In a retrospective study, 12 patients with MPO-ANCA/UIP were compared with $108 \mathrm{IPF} /$ UIP patients [11]. Despite the limitation of the study, the authors did not observe differences between the two populations, including survival and frequency of acute exacerbations [11]. Our results confirm that the radiologic ILD pattern is the best predictor of survival; in particular, in patients with UIP pattern, the presence of anti-MPO did not modify survival when compared with patients with IPF.

Recently, the presence of MPA has been reported as a cause of worst prognosis in patients with interstitial pneumonia and anti-MPO antibodies [31]. The exclusion of patients with a previous diagnosis of vasculitis and the low number of patients that developed a MPA does not allow us to evaluate this point.

The main limitation of our study is its retrospective design; furthermore, five patients were lost at follow-up reducing the number of the evaluated patients. However, this is one of the largest studies investigating this specific population. Moreover, our control group included only Italian IPF patients; although no data were reported in literature about possible differences in clinical history or survival of Spanish and Italian IPF patients, we cannot completely exclude a possible bias. Our results confirm the need to always evaluate 
patients with idiopathic interstitial pneumonia for ANCA, not only at the beginning of the study but also during follow-up. On the other hand, the heterogeneity in the treatment choices highlights the necessity of a consensus for the management of this specific population. Finally, at baseline, three patients satisfied research criteria for IPAF, but antiMPO antibodies are not currently included in the serologic domain of classification IPAF criteria $[17,18]$. The possible evolution in a definite rheumatic disease and the unresolved questions about the treatment of these patients should be taken into account in a future revision of the IPAF criteria, evaluating the inclusion of anti-MPO antibodies.

\section{Conclusions}

Anti-MPO-ILD patients are at risk of progression in a definite rheumatic disease, mainly ANCA-associated vasculitis, in a variable range of time. Therefore, their followup should always include a careful clinical-laboratorial evaluation exploring rheumatic disorders. Only prospective longitudinal studies including a large number of patients may allow us to identify possible associated factors to the evolution towards vasculitis or other inflammatory rheumatic diseases and the prognostic role of anti-MPO antibodies in patients with idiopathic interstitial pneumonia.

Author Contributions: Conceptualization, M.S., F.L., and A.M.; methodology, M.S. and G.S.; software, M.S.; validation, M.S., D.C.V., S.C., and A.M.; formal analysis, M.S. and G.C.; investigation, M.B. and B.A.-M.; resources, A.M. and P.C.; data curation, P.M.A., P.T. and P.F.; writing—original draft preparation, M.S. and E.B.; writing-review and editing, M.S., A.M., F.L., C.V., M.A.G.-G., E.C., and C.S.; supervision, C.S., C.V., M.A.G.-G., and E.C. All authors have read and agreed to the published version of the manuscript.

Funding: This research received no external funding.

Institutional Review Board Statement: The study was conducted according to the guidelines of the Declaration of Helsinki, and approved by the Ethic Committee of Modena.

Informed Consent Statement: Patient consent was waived due to the retrospective design of the study.

Data Availability Statement: Data are available if requested.

Conflicts of Interest: The authors declare no conflict of interest.

\section{References}

1. Falk, R.J.; Jennette, J.C. Anti-Neutrophil Cytoplasmic Autoantibodies with Specificity for Myeloperoxidase in Patients with Systemic Vasculitis and Idiopathic Necrotizing and Crescentic Glomerulonephritis. N. Engl. J. Med. 1988, 318, $1651-1657$. [CrossRef] [PubMed]

2. Jennette, J.C.; Falk, R.J.; Bacon, P.A.; Basu, N.; Cid, M.C.; Ferrario, F.; Flores-Suarez, L.F.; Gross, W.L.; Guillevin, L.; Hagen, E.C.; et al. 2012 Revised International Chapel Hill Consensus Conference Nomenclature of Vasculitides. Arthritis Rheum. 2013, 65, 1-11. [CrossRef]

3. Arimura, Y.; Minoshima, S.; Tanaka, U.; Fujii, A.; Kobayashi, M.; Nakabayashi, K.; Kitamoto, K.; Nagasawa, T. Pulmonary involvement in patients with myeloperoxidase specific-antineutrophil cytoplasmic antibody. Ryumachi 1995, 35, 46-55.

4. Sebastiani, M.; Manfredi, A.; Vacchi, C.; Cassone, G.; Faverio, P.; Cavazza, A.; Sverzellati, N.; Salvarani, C.; Luppi, F. Epidemiology and management of interstitial lung disease in ANCA-associated vasculitis. Clin. Exp. Rheumatol. 2020, 38, 221-231.

5. Alba, M.A.; Flores-Suárez, L.F.; Henderson, A.G.; Xiao, H.; Hu, P.; Nachman, P.H.; Falk, R.J.; Jennette, J.C. Interstital lung disease in ANCA vasculitis. Autoimmun. Rev. 2017, 16, 722-729. [CrossRef]

6. Liu, G.Y.; Ventura, I.B.; Achtar-Zadeh, N.; Elicker, B.M.; Jones, K.D.; Wolters, P.J.; Collard, H.R.; Adegunsoye, A.; Strek, M.E.; Ley, B. Prevalence and Clinical Significance of Antineutrophil Cytoplasmic Antibodies in North American Patients with Idiopathic Pulmonary Fibrosis. Chest 2019, 156, 715-723. [CrossRef]

7. Hozumi, H.; Oyama, Y.; Yasui, H.; Suzuki, Y.; Kono, M.; Karayama, M.; Furuhashi, K.; Enomoto, N.; Fujisawa, T.; Inui, N.; et al. Clinical significance of myeloperoxidase-anti-neutrophil cytoplasmic antibody in idiopathic interstitial pneumonias. PLoS ONE 2018, 13, e0199659. [CrossRef] [PubMed]

8. Ando, M.; Miyazaki, E.; Ishii, T.; Mukai, Y.; Yamasue, M.; Fujisaki, H.; Ito, T.; Nureki, S.-I.; Kumamoto, T. Incidence of myeloperoxidase anti-neutrophil cytoplasmic antibody positivity and microscopic polyangitis in the course of idiopathic pulmonary fibrosis. Respir. Med. 2013, 107, 608-615. [CrossRef] 
9. Kang, B.H.; Park, J.K.; Roh, J.H.; Song, J.W.; Lee, C.K.; Kim, M.; Jang, S.J.; Colby, T.V.; Kim, D.S. Clinical Significance of Serum Autoantibodies in Idiopathic Interstitial Pneumonia. J. Korean Med. Sci. 2013, 28, 731-737. [CrossRef] [PubMed]

10. Nozu, T.; Kondo, M.; Suzuki, K.; Tamaoki, J.; Nagai, A. A Comparison of the Clinical Features of ANCA-Positive and ANCANegative Idiopathic Pulmonary Fibrosis Patients. Respiration 2009, 77, 407-415. [CrossRef]

11. Hosoda, C.; Baba, T.; Hagiwara, E.; Ito, H.; Matsuo, N.; Kitamura, H.; Iwasawa, T.; Okudela, K.; Takemura, T.; Ogura, T. Clinical features of usual interstitial pneumonia with anti-neutrophil cytoplasmic antibody in comparison with idiopathic pulmonary fibrosis. Respirology 2016, 21, 920-926. [CrossRef]

12. Lynch, D.A.; Sverzellati, N.; Travis, W.D.; Brown, K.K.; Colby, T.V.; Galvin, J.R.; Goldin, J.G.; Hansell, D.M.; Inoue, Y.; Johkoh, T.; et al. Diagnostic criteria for idiopathic pulmonary fibrosis: A Fleischner Society White Paper. Lancet Respir. Med. 2018, 6, 138-153. [CrossRef]

13. Tanaka, N.; Kim, J.S.; Newell, J.D.; Brown, K.K.; Cool, C.D.; Meehan, R.; Emoto, T.; Matsumoto, T.; Lynch, D.A. Rheumatoid Arthritis-related Lung Diseases: CT Findings. Radiology 2004, 232, 81-91. [CrossRef]

14. Travis, W.D.; Hunninghake, G.; Talmadge, E.K., Jr.; Lynch, D.A.; Colby, T.V.; Galvin, J.R.; Brown, K.K.; Chung, M.P.; Cordier, J.-F.; Du Bois, R.M.; et al. Idiopathic Nonspecific Interstitial Pneumonia. Am. J. Respir. Crit. Care Med. 2008, 177, 1338-1347. [CrossRef]

15. Walsh, S.L.F.; Sverzellati, N.; Devaraj, A.; Keir, G.J.; Wells, A.U.; Hansell, D.M. Connective tissue disease related fibrotic lung disease: High resolution computed tomographic and pulmonary function indices as prognostic determinants. Thorax 2014, 69, 216-222. [CrossRef] [PubMed]

16. Altman, D. Practical Statistics for Medical Research, 1st ed.; Chapman and Hall: London, UK, 1991.

17. Sebastiani, M.; Cassone, G.; De Pasquale, L.; Cerri, S.; Della Casa, G.; Vacchi, C.; Luppi, F.; Salvarani, C.; Manfredi, A. Interstitial pneumonia with autoimmune features: A single center prospective follow-up study. Autoimmun. Rev. 2020, 19, 102451. [CrossRef] [PubMed]

18. Fischer, A.; Antoniou, K.M.; Brown, K.K.; Cadranel, J.; Corte, T.J.; Du Bois, R.M.; Lee, J.S.; Leslie, K.O.; Lynch, D.A.; Matteson, E.L.; et al. An official European Respiratory Society/American Thoracic Society research statement: Interstitial pneumonia with autoimmune features. Eur. Respir. J. 2015, 46, 976-987. [CrossRef] [PubMed]

19. Becker-Merok, A.; Nossent, J.C.; Ritland, N. Fibrosing alveolitis predating microscopic polyangiitis. Scand. J. Rheumatol. 1999, 28, 254-256. [CrossRef] [PubMed]

20. Kwon, M.; Lee, A.S.; Mira-Avendano, I.; Rojas, C.A.; Grage, R.; Abril, A. Interstitial Lung Disease in Antineutrophil Cytoplasmic Antibody-Associated Vasculitis Patients. JCR J. Clin. Rheumatol. 2020. [CrossRef]

21. Borie, R.; Crestani, B. Antineutrophil Cytoplasmic Antibody-Associated Lung Fibrosis. Semin. Respir. Crit. Care Med. 2018, 39, 465-470. [CrossRef]

22. Katsumata, Y.; Kawaguchi, Y.; Yamanaka, H. Interstitial Lung Disease with ANCA-associated Vasculitis. Clin. Med. Insights Circ. Respir. Pulm. Med. 2015, 9 (Suppl. 1), 51-56. [CrossRef] [PubMed]

23. Casares, M.F.; Gonzalez, A.; Fielli, M.; Caputo, F.; Bottinelli, Y.; Zamboni, M. Microscopic polyangiitis associated with pulmonary fibrosis. Clin. Rheumatol. 2014, 34, 1273-1277. [CrossRef]

24. Arulkumaran, N.; Periselneris, N.; Gaskin, G.; Strickland, N.; Ind, P.W.; Pusey, C.D.; Salama, A.D. Interstitial lung disease and ANCA-associated vasculitis: A retrospective observational cohort study. Rheumatology 2011, 50, 2035-2043. [CrossRef]

25. Flores-Suárez, L.F.; Ruiz, N.; Rivera, L.M.S.; Pensado, L. Reduced survival in microscopic polyangiitis patients with pulmonary fibrosis in a respiratory referral centre. Clin. Rheumatol. 2015, 34, 1653-1654. [CrossRef] [PubMed]

26. Kagiyama, N.; Takayanagi, N.; Kanauchi, T.; Ishiguro, T.; Yanagisawa, T.; Sugita, Y. Antineutrophil cytoplasmic antibody-positive conversion and microscopic polyangiitis development in patients with idiopathic pulmonary fibrosis. BMJ Open Respir. Res. 2015, 2, e000058. [CrossRef]

27. Tur, B.S.; Süldür, N.; Ataman, S.; Tutkak, H.; Atay, M.B.; Düzgün, N. Anti-neutrophil cytoplasmic antibodies in patients with rheumatoid arthritis: Clinical, biological, and radiological correlations. Jt. Bone Spine 2004, 71, 198-202. [CrossRef]

28. Guellec, D.; Gall, E.C.-L.; Groh, M.; Hachulla, E.; Karras, A.; Charles, P.; Dunogué, B.; Abad, S.; Alvarez, F.; Gérard, F.; et al. ANCA-associated vasculitis in patients with primary Sjögren's syndrome: Detailed analysis of 7 new cases and systematic literature review. Autoimmun. Rev. 2015, 14, 742-750. [CrossRef] [PubMed]

29. Merkel, P.A.; Polisson, R.P.; Chang, Y.; Skates, S.J.; Niles, J.L. Prevalence of antineutrophil cytoplasmic antibodies in a large inception cohort of patients with connective tissue disease. Ann. Intern. Med. 1997, 126, 866-873. [CrossRef]

30. Maillet, T.; Goletto, T.; Beltramo, G.; Dupuy, H.; Jouneau, S.; Borie, R.; Crestani, B.; Cottin, V.; Blockmans, D.; Lazaro, E.; et al. Usual interstitial pneumonia in ANCA-associated vasculitis: A poor prognostic factor. J. Autoimmun. 2020, 106, 102338. [CrossRef]

31. Sun, X.; Peng, M.; Zhang, T.; Li, Z.; Song, L.; Li, M.; Shi, J. Clinical features and long-term outcomes of interstitial lung disease with anti-neutrophil cytoplasmic antibody. BMC Pulm. Med. 2021, 21, 88. [CrossRef] [PubMed] 